RED. Revista de Educación a Distancia. Núm. 51. Artic. 6. 15-11-2016

DOI: http://dx.doi.org/10.6018/red/51/6

http://www.um.es/ead/red/islas.pdf

\title{
Representaciones sociales de estudiantes universitarios acerca del b-learning: un análisis de contenido
}

\section{Social representations of university students about b-learning: a content analysis}

\author{
Claudia Islas \\ Universidad de Guadalajara. México \\ cislas@ cualtos.udg.mx
}

\begin{abstract}
Resumen
Actualmente el b-learning es considerado como una de las modalidades de enseñanzaaprendizaje más prometedoras, esto por la flexibilidad de estrategias y acciones que permite articular desde lo presencial y virtual. Bajo este supuesto, se presentan los resultados de una investigación de tipo cualitativo, cuyo objetivo fue conocer las representaciones que estudiantes universitarios construyen a partir de su vivencia cursando asignaturas de licenciatura en esta modalidad. La información deriva de la técnica de comparación constante y el método de análisis de contenido aplicado a 18 entrevistas a profundidad, dirigidas a estudiantes de una Universidad Pública del estado de Jalisco, México. Los resultados, interpretados desde la Teoría de las Representaciones Sociales dan indicios de que ellos elaboran opiniones a partir de sus creencias y percepciones, contacto e interacción con el profesor, así como desde las habilidades que desarrollan para adaptarse a la modalidad y lo que ésta les implica.
\end{abstract}

\section{Palabras clave}

Análisis de contenido, b-learning, Estudiantes universitarios, Representaciones sociales, Tecnologías.

\begin{abstract}
Currently, b-learning is regarded as one of the most promising styles of teaching and learning. This is due to the flexibility of strategies and actions that can be implemented in the classroom and in a virtual environment. Under this assumption, the results are demonstrated by a qualitative study whose objective was to show the representations that university students built from their experience of studying certain majors within this field. The information is from the constant comparison technique and content analysis method applied to 18 in-depth interviews, aimed at students of a public university in the state of Jalisco, Mexico. The results, interpreted from the Theory of Social Representations, show evidence that they produce views from their beliefs and perceptions, contact and interaction with the teacher, as well as from the skills they develop and what it implies to adapt with the method.
\end{abstract}

\section{Keywords}

Content analysis, b-learning, social representations, university students, technologies. 


\section{Introducción}

El hecho de que la incursión de las Tecnologías de la Información y Comunicación (TIC) transformaron el ámbito de la educación superior es innegable, en la literatura de la última década se identifican numerosas publicaciones que hablan del impacto (ÁvilaFajardo y Riascos-Erazo, 2013; Chiecher, Donolo, y Rinaudo, 2010; Claro, 2010; Tello, 2008), la implementación de cursos mediados por TIC (Carcaño y Torres, 2013; Ojeda, Uicab, y Aguilar, 2011; Pulido y Pulido, 2012), así como la conceptualización que se le da a la diversidad de modalidades que se gestan a partir del uso de tecnologías, tales como e-learning (aprendizaje electrónico ó en línea), u-learning (aprendizaje universal), m-learning (aprendizaje móvil), b-learning (aprendizaje mezclado), entre otras (Ferreiro y DeNapoli, 2006); las competencias que desarrollan los estudiantes universitarios (Arras, Torres, y García-Valcárcel, 2011; Villanueva y De la Luz, 2010; Viñas, 2014) y varios aspectos más, que dan cuenta de la relevancia de las TIC en los procesos formativos.

En este sentido, las modalidades de enseñanza-aprendizaje emergentes a partir de la implementación de tecnologías, así como todo lo que se asocia a ellas, se estudia desde distintos puntos de vista, referentes teóricos, métodos, visiones y objetivos; por lo que puede encontrarse información de diversa índole que alimenta el cuerpo de conocimiento que se genera tras las inquietudes de los investigadores y expertos en el área.

Es así, que el presente documento describe los resultados recuperados en una investigación cualitativa, dirigida a conocer las representaciones que estudiantes universitarios elaboran a partir de su vivencia cursando asignaturas de licenciatura en la modalidad b-learning, escenario que se configura tras la mezcla de acciones presenciales y virtuales en las que estudiantes, docentes y contenidos, interactúan construyendo un ambiente educativo propicio para la enseñanza y el aprendizaje.

En el documento se exponen los antecedentes de esta investigación, detalles conceptuales respecto a la modalidad, el referente teórico del que se partió para hacer el planteamiento y la interpretación de los datos, y las técnicas y método aplicados para el análisis de la información obtenida a través de entrevistas a profundidad aplicadas a 18 estudiantes de licenciatura de una Universidad Pública del estado de Jalisco, México.

\section{Antecedentes y marco referencial}

La literatura publicada en la última década respecto a los ambientes de enseñanzaaprendizaje mixtos o también denominados b-learning expresa desde distintas posturas y enfoques cómo se ha percibido y apropiado esta modalidad de formación por parte de estudiantes, docentes e instituciones (Islas, 2014), cómo se opera, cómo es definida (Turpo, 2009) o conceptualizada por distintos investigadores que tras varios años han dedicado parte de sus estudios a describir y explicar esta modalidad.

A partir del análisis de distintas publicaciones se identificó, que a través de la teoría de las representaciones sociales podría contribuirse al cuerpo de conocimiento que se ha gestado en los últimos años en torno a lo que los estudiantes viven o experimentan cuando se enfrentan a escenarios mezclados entre presencialidad y virtualidad. El reflejo de la realidad que emerge al aplicar dicha teoría en conjunto con la técnica de comparación constante y el análisis de contenido, permitió acercarse a la construcción

Representaciones sociales de estudiantes universitarios acerca del b-learning: un análisis de contenido. Claudia Islas Torres

Página 2 | 19 
subjetiva que los estudiantes cimentan como eje conductor de su actuar cuando se ven inmersos en un ambiente educativo no convencional.

\section{B-learning: su conceptualización y estado del arte}

El aprendizaje mezclado o b-learning se concibe como una combinación de actividades tradicionales en el aula y actividades en línea que integra las tecnologías Web en el proceso de enseñanza-aprendizaje, mezcla que posibilita la implementación de distintos métodos pedagógicos y estrategias de enseñanza que pueden trascender los límites de las aulas y las aplicaciones mismas (Peñalosa, García, Martínez, y Rojas, 2010), esta definición coincide con la de Valiathan (2002) quien conceptualizó esta modalidad como la mezcla de varios eventos que incluyen las clases cara a cara en combinación con el e-learning. Por su parte, Bartolomé (2004) la concibió como una forma de aprender que combina la enseñanza presencial con la tecnología no presencial, donde la clave es la selección de medios adecuados a cada necesidad educativa.

Desde la perspectiva de Garrison y Kanuka (2004), el b-learning debe entenderse como una forma renovada de plantear y pensar la relación entre la enseñanza y el aprendizaje, puesto que lo anterior implica desafíos donde se requiere de entornos que transformen las habilidades de quienes ahí interactúan para potenciar el pensamiento crítico, creativo y complejo.

Rama (2007), Silvio (sf), Turpo (2012) y Vasileiou (2009) coinciden en definir al $b$ learning como un modelo de educación que mezcla elementos presenciales y virtuales que conducen a una despresencialización de la educación, integrando diversas metodologías pedagógicas y medios tecnológicos que propician la construcción del conocimiento.

Esta modalidad de formación se destaca por los beneficios que representa, puesto que combina lo mejor del e-learning y el aprendizaje presencial, da flexibilidad para la realización de actividades en el tiempo y el espacio, facilitando la interacción y oportunidades de participación, así como el seguimiento a las acciones de los estudiantes y la retroalimentación requerida por ellos.

Los beneficios del b-learning identificados a lo largo de los años se han reflejado en múltiples publicaciones que hablan de su implementación, de las experiencias, la valoración, la calidad, entre otros aspectos; como lo que presentan Esparaza, Salinas y Glasserman (2015) quienes describieron cómo la gestión del aprendizaje en esta modalidad posibilita el desarrollo de habilidades gramaticales inglesas en estudiantes de preparatoria, tras aplicar una metodología cuantitativa con un diseño cuasiexperimental los resultados indican que los estudiantes que participaron en la modalidad mixta cometieron menos errores en la redacción, lo cual confirmó que ésta es una estrategia factible para el aprendizaje de ésta gramática.

Una experiencia más, respecto a la implementación del b-learning en la enseñanza de lengua inglesa es reportada por Castañeda y López (2014) quienes encontraron a través de encuestas y entrevistas directas que para los estudiantes esta modalidad es satisfactoria en el logro de su aprendizaje, pero también se toparon con desventajas y limitaciones debido a que algunos de ellos no tenían experiencia sobre el uso de las TIC.

Peres, Lima y Lima (2014) presentaron una colección de criterios que permiten valorar un ambiente b-learning entre los que destacan aspectos propios del curso, así como

Representaciones sociales de estudiantes universitarios acerca del b-learning: un análisis de contenido. 
institucionales, de diseño, desarrollo, implementación y evaluación, y cuestiones de tipo pedagógicas, con el fin de orientar sobre las instrucciones que llevarían al logro del aprendizaje de los estudiantes.

El b-learning también se ha considerado como ambiente educativo propicio para el desarrollo de aprendizajes basados en problemas, tal es el caso que describen Trujillo, Hinojo, Marín, Romero, y Campos (2014) quienes refieren que éste tipo de aprendizajes brindan una oportunidad para la comunicación, colaboración, compromiso, innovación reflexiva y crítica que puede sustentarse en ambientes del tipo mixto donde se da significatividad al conocimiento adquirido a través de la interacción efectiva.

Lynch (2014) presentó un estudio de tipo cualitativo en el que trató de rescatar como los estudiantes universitarios que se involucran en ambientes b-learning valoran la flexibilidad que les da la comunicación síncrona y asíncrona, dando apertura al diseño de estructuras deliberadas que les ayuda a hacer uso de un espacio configurable a sus necesidades.

La percepción de los estudiantes respecto a esta modalidad ha sido estudiada por algunos autores y sus hallazgos reportan una preferencia marcada hacia lo mixto, ya que éstos encuentran ventajas como la flexibilidad horaria, en las metodologías de enseñanza, en el acceso a la información, rapidez en la comunicación, entre otras. (Hinojo y Aznar, 2009; Ruíz, 2008; Miyazoe y Anderson, 2010)

Uno más de los aspectos considerados en las publicaciones sobre b-learning hace referencia a las bondades y características de la plataforma tecnológica Moodle, misma que se utiliza para configurar y gestionar los cursos que se habilitan, para satisfacer necesidades de estudiantes y docentes. (Pulido y Pulido, 2012)

Por su parte Mortis, Hierro, García y Manig (2015) presentaron un estudio cuantitativocualitativo en el que a través del interaccionismo simbólico se analizó lo que significa para los estudiantes el participar en un curso de modalidad mixta, los resultados mostraron que éstos valoran positivamente dichos cursos y sugieren mejoras en la planeación de actividades, la comunicación y retroalimentación del docente.

Como puede observarse en los párrafos anteriores, el acercamiento al estudio de lo que sucede en los cursos b-learning se hace desde diversas perspectivas y posturas, por lo que a continuación se expone el referente epistémico que para efectos de esta investigación permitió plantear e interpretar los resultados.

\section{La Teoría de las Representaciones Sociales y su aplicabilidad al estudio de fenómenos educativos}

Ante la necesidad de encontrar un referente teórico que diera cuenta de lo que los estudiantes universitarios expresan a partir de sus vivencias cuando cursan asignaturas en modalidades no convencionales, y todo lo que a ellos les representa o significa, se encontró que la Teoría de las Representaciones Sociales es un marco epistémico que desde la perspectiva de Moscovici permite dar inteligibilidad a las relaciones cotidianas de intercambios e imaginación (como se citó en Mora, 2002). Las representaciones sociales acercan al conocimiento de los comportamientos cotidianos que son inducidos por las representaciones personales que cada cual tiene sobre una realidad, mismas que se convierten en una guía generadora de comportamientos. (Lobato, del Castillo, y Arbizu, 2005)

Representaciones sociales de estudiantes universitarios acerca del b-learning: un análisis de contenido. Claudia Islas Torres 
La Teoría de las Representaciones Sociales se encuentra en desarrollo y debate, esta surge en Francia en la década de los 60's y se ha utilizado como referente en investigaciones empíricas de orden interdisciplinario por su aplicabilidad como un planteamiento metodológico para el análisis de lo cotidiano y común, dando espacio a la construcción social de la realidad. (Materán, 2008)

A decir de Moscovici, las representaciones sociales son una expresión cognitiva, construida con una lógica y lenguaje propios, donde se integra un conjunto de creencias, valores e ideas que permiten a los individuos orientarse en su realidad y generar conocimiento que incide en su pensamiento y en la organización de su cotidianeidad (como se citó en Mora, 2002). Las representaciones son una forma concreta de comunicar y comprender lo que se conoce, de predecir comportamientos y de analizar conductas. En el ámbito de la educación, éstas, han servido para conocer el comportamiento de docentes y estudiantes ante ciertas situaciones, llevando a deducir que las creencias, interacciones o percepciones, son incidentes en la práctica de quienes se involucran en los procesos formativos. (Mora, 2002)

Por tanto, las representaciones permiten transitar de la elaboración de conceptos a la construcción de teorías, puesto que se constituyen en una nueva unidad de enfoque que integra lo individual y colectivo; lo simbólico y social; y el pensamiento y la acción. (Chourio, 2012)

En este sentido, la utilidad de las representaciones sociales se concibe ante la posibilidad de sintetizar las explicaciones que las personas hacen en torno a su realidad, a lo que piensan y viven, beneficio que para esta investigación fue tomado en cuenta, puesto que como referente permitió extractar lo que los estudiantes exponen en torno a su vivencia en una modalidad b-learning.

De lo anterior se infiere que las representaciones sociales permiten formar conocimiento que reconoce los estereotipos, opiniones, creencias, valores y normas que llevan a los individuos a actuar de forma positiva o negativa. Las mismas pueden servir como sistemas de código, valores, lógicas clasificatorias, principios interpretativos y orientadores sobre lo que define la consciencia colectiva y lo que les da apertura o limitaciones para actuar (Chourio, 2012). En ese sentido, estas representaciones acercan a conocer cómo los estudiantes se desempeñan en una modalidad a partir de lo que ellos creen, perciben o socializan con los demás, guiando así su actuar en el ambiente formativo.

Una manera más de entender las representaciones sociales, es la expresada por Acosta y Uribe, quienes aludieron a la doble modalidad que éstas permiten, es decir, pueden considerarse como una forma activa de reproducción de las características de un objeto; de su reconstrucción mental, o como la forma de pensamiento social que estructura la comunicación y las conductas de los miembros de grupos (como se citó en Mora, 2002).

Por su parte León (como se citó en Materán, 2008) y Darío Páez (como se citó en Mora, 2002) dieron una caracterización de las representaciones sociales aludiendo a cinco puntos esenciales:

1. Dar sentido o forma definitiva a los objetos, personas o eventos que se encuentran en la cotidianeidad para que éstos pasen de una realidad extraña a una familiar.

Representaciones sociales de estudiantes universitarios acerca del b-learning: un análisis de contenido. 
2. Promover la comunicación entre las personas dando cabida a todos los puntos de vista que puedan surgir ante diversas cuestiones.

3. Descomponer los rasgos en categorías simples estableciendo y objetivando los conceptos que surgen del discurso que pueden expresar los sujetos.

4. Generar un modelo sintético o teoría implícita, explicativa y evaluativa del entorno a partir del discurso que impregna al sujeto.

5. El proceso reconstruye y reproduce la realidad otorgándole un sentido y procura una guía operacional para la vida social, para la resolución de los problemas y conflictos.

Estos puntos característicos de las representaciones sociales guiaron las acciones que se aplicaron en esta investigación, puesto que se consideró que podían seguirse para reproducir las características de las vivencias de los estudiantes al involucrarse en ambientes formativos del tipo b-learning.

Bajo este supuesto, y en términos de la investigación que aquí se describe, se corroboró que una de las funciones de la teoría de las representaciones sociales es dar sentido y explicar la realidad como un saber práctico que permite a los actores sociales adquirir conocimientos e integrarlos en un marco asimilable y comprensible para transmitir o comunicar. (Abric, 1994)

\section{Enfoque y método de investigación}

La investigación que aquí se reporta corresponde a un estudio de tipo cualitativo (Hernández, Fernández y Baptista, 2010) en el que la fortaleza para hacerse de información fue el método de entrevistas a profundidad (Kvale, 2011) a través de las cuales se intentó entender los asuntos cotidianos de los estudiantes que vivían la experiencia de participar en un curso de modalidad mixta. Las entrevistas a través del lenguaje normal buscan conocimiento cualitativo acercándose a relatos matizados con diferentes aspectos que dan significado de relevancia para el investigador, quien trata la información cuidando mantener la perspectiva y palabras del informante respecto a la situación que narra desde su vivencia, destacando lo que dice y cómo lo dice.

Tomando las consideraciones anteriores se aplicó como primer método un análisis de contenido (López, 2002) a 18 entrevistas a profundidad, aplicadas a estudiantes de licenciatura de una Universidad Pública del estado de Jalisco.

Las entrevistas originaron una cantidad de información importante y que a primera vista parecía desorganizada, por lo que había que descomponerla en un conjunto de rasgos y categorías que permitieran darle sentido y objetividad.

Las entrevistas se estructuraron bajo preguntas abiertas que daban oportunidad para que los estudiantes expresaran sus vivencias al cursar asignaturas en dicha modalidad, conforme se realizaba una entrevista se procedía a la captura de la misma, para así tener como base información textual que al organizarla y categorizarla sirviera para rescatar las ideas expresadas en el discurso de los estudiantes y poder cuantificarlas dependiendo del significado o tema que abordaran.

Los datos se codificaron utilizando el método de comparación constante desde la Teoría Fundamentada (Strauss y Corbin, 2002). Este procedimiento de análisis permitió

Representaciones sociales de estudiantes universitarios acerca del b-learning: un análisis de contenido. 
comparar las entrevistas y así jerarquizar la información, con la intención de determinar cuál era el núcleo central de las representaciones y sobre qué se estructuraron, así, los datos se agruparon en conjuntos homogéneos que llevaron a establecer relaciones e inferencias entre los diversos temas analizados y de éstos con teorías previas puesto que la teoría permite la generación de conceptos asociados a lo que se va encontrando en el estudio.

Partiendo del supuesto de que la teoría fundamentada ayudaría a conocer las representaciones que los estudiantes construyen, se procedió a aplicar este método de análisis con el propósito de rescatar el núcleo figurativo que diera claridad al fenómeno observado cargado de subjetividad. (Campo-Redondo y Labarca, 2009)

Por tanto, la teoría fundamentada, permitió reconocer los componentes bajo los cuales los estudiantes estructuran sus representaciones, partiendo desde sus criterios y asociándolos a sus creencias y valores, lo cual garantizó que se usara con fidelidad lo expresado por ellos manteniendo así la significatividad de sus palabras.

El análisis de contenido partió de la definición previa de dimensiones (cognitiva, actitudinal, aprendizaje, tecnológica y comunicativa) que sirvieron para describir cuantitativamente las acciones de los estudiantes universitarios cuando se ven inmersos en una modalidad b-learning (Islas, 2015). Ese primer acercamiento permitió que para el análisis de contenido se tuviesen presentes los supuestos desde los cuales se inició el trabajo de investigación, y al mismo tiempo dio indicios de las relaciones que surgían en los discursos de las entrevistas y las subcategorías que podían formarse a partir de ellas. El estudio de datos se llevó a cabo de acuerdo al proceso de análisis cualitativos descrito por (Lobato, del Castillo, y Arbizu, 2005) que se presenta en la figura 1.

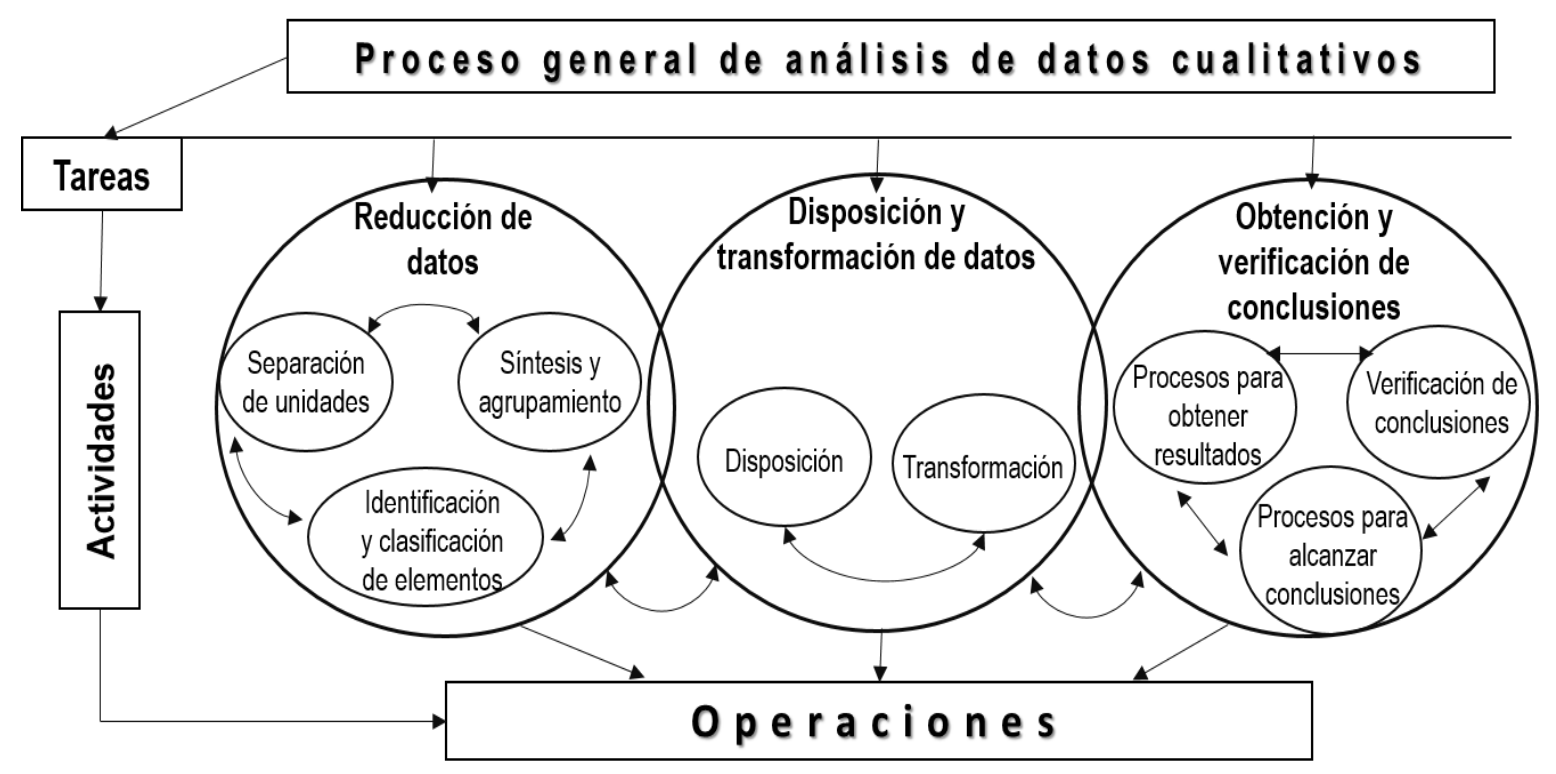

Figura 1. Proceso de análisis de datos cualitativos. Fuente: (Lobato, del Castillo, y Arbizu, 2005)

Considerando que el análisis de contenido permite hacer una descripción objetiva, sistemática y cuantitativa del contenido que se manifiesta en un discurso, éste se llevó a cabo utilizando el software Atlas.Ti versión 7, herramienta que facilitó la categorización, codificación y conteo de frecuencia de unidades textuales que a su vez fueron los indicadores que explicaron el fenómeno estudiado.

Representaciones sociales de estudiantes universitarios acerca del b-learning: un análisis de contenido. Claudia Islas Torres

Página 7 | 19 


\section{Informantes clave}

Bajo el supuesto de que en una investigación cualitativa el tamaño de la muestra no es importante desde una perspectiva probabilística (Hernández, Fernández, y Baptista, 2010) porque lo que se pretende es una indagación a profundidad, se buscó a estudiantes que hubieran cursado al menos una asignatura en modalidad $b$-learning y que tuvieran disponibilidad para participar accediendo a ser entrevistados, por lo que la muestra fue autoseleccionada y a conveniencia del investigador.

Los estudiantes entrevistados fueron 18, de los cuales, 5 estaban inscritos en la licenciatura en Administración, 3 en Negocios Internacionales, 6 en Ingeniería en Computación y 4 en Contaduría. Respecto al género de los participantes 9 son Hombres y 9 Mujeres.

\section{Resultados}

A continuación se describen los resultados obtenidos tras la etapa de análisis que comprendió la organización de los datos, en ella se procuró colocar toda la información dentro de categorías conceptuales, a cada una de éstas se le asignó un nombre en términos de la interpretación de lo que se infirió estaba expresando el discurso analizado; así, se corroboró que la clasificación inicial para representar cuantitativamente (Islas, 2015) coincidía con lo que ahora a través del discurso se vislumbraba. De igual manera, el nombrar las categorías y sus códigos permitió captar la complejidad de la relación que se da entre las ideas y los significados que implican. En este sentido, emergieron los elementos que explicaron los sucesos y que al mismo tiempo se extraían del lenguaje de los informantes.

Posteriormente se hizo una comparación entre categorías con sus respectivos códigos de tal forma que se pudiera clarificar dónde había coincidencias y divergencias en los discursos y en la información clasificada, así se rescató todo aquello que refiriera a la vivencia de los estudiantes al cursar asignaturas en la modalidad mencionada. Esta etapa representó el trabajo de organización y jerarquización de los contenidos reduciéndose a categorías principales.

Los códigos emergentes se delimitaron en función del marco teórico que acompañó a la investigación, en este caso la conceptualización de la modalidad y los antecedentes empíricos de otras investigaciones, con la intención de clarificar lo que los estudiantes contaban acerca del fenómeno, con ello pudo eliminarse todo lo que parecía redundante.

Una vez que se tenían las categorías, sus códigos y conceptualizaciones asociadas, se volvió a releer la información textual con la finalidad de identificar la saturación teórica (Campo-Redondo y Labarca, 2009) que surge cuando se observa que los discursos son repetitivos y que corresponden a las clasificaciones establecidas y validadas.

A partir de los pasos previos, a continuación se exponen las categorías estructuradas a partir de las representaciones sociales que poseen los estudiantes acerca de su experiencia al cursar asignaturas en modalidad b-learning. El análisis de contenido dio cuenta de los factores que se vinculan a esta modalidad y de cómo los estudiantes se ubican en el ambiente que se genera. La tabla 1 muestra las categorías, los códigos derivados y las conceptualizaciones, así mismo, integra lo significativo de los discursos que se obtuvo desde la comparativa constante.

Representaciones sociales de estudiantes universitarios acerca del b-learning: un análisis de contenido. 
Tabla 1. Sistema de categorías

\begin{tabular}{|c|c|c|}
\hline Categorías & Códigos & Conceptualización \\
\hline 1. Cognición & $\begin{array}{l}\text { 1.1 Percepción } \\
\text { 1.1.1 Sobre el } \\
\text { profesor } \\
\text { 1.1.2 Métodos de } \\
\text { evaluación } \\
\text { 1.2 Razonamiento } \\
\text { 1.2.1 Esfuerzo y } \\
\text { dedicación } \\
\text { 1.2.2 Habilidad } \\
\text { para resolver } \\
\text { problemas }\end{array}$ & $\begin{array}{l}\text { La cognición son las estructuras mentales y } \\
\text { conocimientos que el estudiante aplica para } \\
\text { el logro de su aprendizaje o para la } \\
\text { elaboración conceptual de las situaciones } \\
\text { que experimenta. } \\
\text { Sus discursos hablan de su percepción } \\
\text { respecto al profesor y su práctica, y de los } \\
\text { métodos de evaluación aplicados a su acción } \\
\text { como estudiantes. } \\
\text { Lo cognitivo también implica el } \\
\text { razonamiento, su esfuerzo y dedicación a las } \\
\text { clases y lo que el b-learning les representa, } \\
\text { así como las habilidades que desarrollan } \\
\text { para resolver problemas. }\end{array}$ \\
\hline 2. Actitud & $\begin{array}{l}\text { 2.1 Disposición al } \\
\text { cambio } \\
2.1 .1 \\
\text { Disponibilidad } \\
\text { al trabajo en } \\
\text { línea } \\
\text { 2.1.2 Organización } \\
\text { del tiempo } \\
\text { 2.1.3 Aceptación } \\
\text { del ambiente } \\
\text { b-learning } \\
\text { 2.1.4 Apertura al } \\
\text { uso de TIC } \\
\text { 2.2 Dependencia } \\
\text { hacia el docente } \\
\text { 2.3 Influye en la } \\
\text { acción del docente }\end{array}$ & $\begin{array}{l}\text { La actitud para efectos de esta investigación } \\
\text { se conceptualizó como el conjunto de } \\
\text { relaciones entre creencias, conductas y } \\
\text { emociones que un sujeto puede tener ante } \\
\text { determinado objeto, en ese sentido, las } \\
\text { actitudes de los estudiantes identificadas van } \\
\text { desde la disponibilidad al cambio, la } \\
\text { dependencia hacia el docente y su influencia } \\
\text { en la acción del docente. }\end{array}$ \\
\hline 3. Aprendizaje & $\begin{array}{l}\text { 3.1 Autogestión } \\
\text { 3.2 Uso de estrategias } \\
\text { 3.3 Colaboración } \\
\text { 3.4 Aprendizaje } \\
\text { significativo } \\
\text { 3.5 Actividades de } \\
\text { aprendizaje } \\
\text { 3.6 Contenidos } \\
\text { acordes a sus } \\
\text { necesidades }\end{array}$ & $\begin{array}{l}\text { El aprendizaje se conceptualizó como la } \\
\text { implicación de procesos en los que los } \\
\text { estudiantes involucran actividades } \\
\text { organizadas de forma responsable que le } \\
\text { conducen a la asimilación y elaboración de } \\
\text { conocimiento. En el aprendizaje destacan } \\
\text { acciones de autogestión, aplicación o uso de } \\
\text { estrategias, colaboración y perspectivas } \\
\text { respecto a las actividades de aprendizaje y } \\
\text { los contenidos que se presentan. }\end{array}$ \\
\hline 4. Tecnología & $\begin{array}{l}\text { 4.1 Respecto a la } \\
\text { plataforma } \\
\text { 4.2 De sus } \\
\text { habilidades } \\
\text { tecnológicas }\end{array}$ & $\begin{array}{l}\text { Definida como las habilidades que los } \\
\text { estudiantes manifiestan respecto al dominio } \\
\text { de tecnologías, así como el uso de éstas en } \\
\text { función de sus objetivos de aprendizaje. } \\
\text { En la categoría de tecnologías destaca su }\end{array}$ \\
\hline
\end{tabular}

Representaciones sociales de estudiantes universitarios acerca del b-learning: un análisis de contenido. 


\begin{tabular}{|c|c|c|}
\hline & $\begin{array}{l}\text { 4.3 Uso de redes } \\
\text { sociales } \\
\text { 4.4 Uso de internet } \\
\text { 4.5 Administración de } \\
\text { la información } \\
\text { 4.6 Empleo de } \\
\text { herramientas web } 2.0\end{array}$ & $\begin{array}{l}\text { referencia respecto a la plataforma Moodle, } \\
\text { sus habilidades tecnológicas, el uso de redes } \\
\text { sociales y de internet, la administración de } \\
\text { información y el empleo de herramientas } \\
\text { web 2.0. }\end{array}$ \\
\hline $\begin{array}{l}5 . \\
\text { Comunicación }\end{array}$ & $\begin{array}{l}\text { 5.1 Retroalimentación } \\
\text { docente } \\
\text { 5.1.1 Oportuna } \\
\text { 5.1.2 Resolución } \\
\text { de dudas } \\
\text { 5.1.3 Sobre la } \\
\quad \text { forma de las } \\
\text { actividades } \\
\text { 5.2 Intercambio de } \\
\text { opiniones entre } \\
\text { compañeros } \\
\text { 5.3 Negociación con } \\
\text { el docente } \\
\text { 5.4 Interpretación de } \\
\text { instrucción }\end{array}$ & $\begin{array}{l}\text { Conceptualizada como uno de los factores } \\
\text { determinantes en un ambiente b-learning, en } \\
\text { la comunicación se enmarca la interacción } \\
\text { con los compañeros y con el mismo docente. } \\
\text { La comunicación es la forma de expresión } \\
\text { que permite retroalimentar, negociar, } \\
\text { intercambiar opiniones y exponer lo que se } \\
\text { cree o percibe. }\end{array}$ \\
\hline
\end{tabular}

Considerando como eje conductor a cada una de las categorías derivadas, que se presentaron en la tabla 1, a continuación (tabla 2) se expone el análisis de las subcategorías de acuerdo a la frecuencia de aparición y el porcentaje que constituye en la totalidad de representaciones.

Tabla 2.Unidades textuales

\begin{tabular}{|c|c|c|c|}
\hline Categorías & Códigos & $\begin{array}{c}\text { Número } \\
\text { total de } \\
\text { apariciones }\end{array}$ & $\begin{array}{l}\text { Porcentaje } \\
\text { que } \\
\text { representan } \\
\text { en la } \\
\text { categoría }\end{array}$ \\
\hline \multirow{6}{*}{$\begin{array}{l}\text { 1. Cognición } \\
\text { Total: } 157 \\
\text { Representa:18\% }\end{array}$} & 1.1 Percepción & 124 & $\mathbf{7 9 \%}$ \\
\hline & 1.1.1 Sobre el profesor & 98 & $62 \%$ \\
\hline & 1.1.2 Métodos de evaluación & 26 & $17 \%$ \\
\hline & 1.2 Razonamiento & 33 & $21 \%$ \\
\hline & 1.2.1 Esfuerzo y dedicación & 22 & $14 \%$ \\
\hline & 1.2.2 Habilidad para resolver problemas & 11 & $7 \%$ \\
\hline \multirow{7}{*}{$\begin{array}{l}\text { 2. Actitud } \\
\text { Total: } 259 \\
\text { Representa:30\% }\end{array}$} & 2.1 Disposición al cambio & 192 & $74 \%$ \\
\hline & 2.1.1 Disponibilidad al trabajo en línea & 74 & $29 \%$ \\
\hline & 2.1.2 Organización del tiempo & 46 & $18 \%$ \\
\hline & 2.1.3 Aceptación del ambiente b-learning & 37 & $14 \%$ \\
\hline & 2.1.4 Apertura al uso de TIC & 35 & $14 \%$ \\
\hline & 2.2 Dependencia hacia el docente & 42 & $16 \%$ \\
\hline & 2.3 Influye en la acción del docente & 25 & $10 \%$ \\
\hline
\end{tabular}

Representaciones sociales de estudiantes universitarios acerca del b-learning: un análisis de contenido. 


\begin{tabular}{|c|c|c|c|}
\hline \multirow{6}{*}{$\begin{array}{l}\text { 3. Aprendizaje } \\
\text { Total: } 154 \\
\text { Representa:18\% }\end{array}$} & 3.1 Autogestión & 53 & $34 \%$ \\
\hline & 3.2 Uso de estrategias & 39 & $25 \%$ \\
\hline & 3.3 Colaboración & 28 & $18 \%$ \\
\hline & 3.4 Aprendizaje significativo & 14 & $9 \%$ \\
\hline & 3.5 Actividades de aprendizaje & 4 & $3 \%$ \\
\hline & 3.6 Contenidos acorde a sus necesidades & 16 & $10 \%$ \\
\hline \multirow{6}{*}{$\begin{array}{l}\text { 4. Tecnología } \\
\text { Total: } 154 \\
\text { Representa:18\% }\end{array}$} & 4.1 Respecto a la plataforma & 4 & $3 \%$ \\
\hline & 4.2 De sus habilidades tecnológicas & 21 & $14 \%$ \\
\hline & 4.3 Uso de redes sociales & 26 & $17 \%$ \\
\hline & 4.4 Uso de internet & 28 & $18 \%$ \\
\hline & 4.5 Administración de la información & 29 & $19 \%$ \\
\hline & 4.6 Empleo de herramientas web 2.0 & 12 & $8 \%$ \\
\hline \multirow{7}{*}{$\begin{array}{l}\text { 5. Comunicación } \\
\text { Total: } 141 \\
\text { Representa: } \\
16 \%\end{array}$} & 5.1 Retroalimentación docente & 59 & $42 \%$ \\
\hline & \begin{tabular}{|l|}
5.1 .1 Oportuna \\
\end{tabular} & 22 & $16 \%$ \\
\hline & 5.1.2 Resolución de dudas & 31 & $22 \%$ \\
\hline & 5.1.3 Sobre la forma de las actividades & 6 & $4 \%$ \\
\hline & 5.2 Intercambio de opiniones entre compañeros & 22 & $16 \%$ \\
\hline & 5.3 Negociación con el docente & 46 & 33\% \\
\hline & 5.4 Interpretación de instrucción & 14 & $10 \%$ \\
\hline $\begin{array}{c}\text { Número total de } \\
\text { unidades } \\
\text { textuales }\end{array}$ & & 865 & \\
\hline
\end{tabular}

Este análisis de contenido permitió recuperar los fragmentos de texto más relevantes y extraer las ideas centrales que sintetizan el contenido de lo que describe a las representaciones de los estudiantes.

Los discursos se analizaron considerando las categorías surgidas de los mismos en el proceso de comparación constante con base al cuestionamiento: ¿Cuál ha sido tu experiencia cursando asignaturas en modalidad b-learning? a partir de sus comentarios se identificó que éstos versaban acerca de lo que se determinó como cognitivo (18\%), haciendo notorios aspectos de su percepción sobre el profesor, representada en un $62 \%$ de apariciones, se refirieron a las habilidades tecnológicas con las que cuenta el docente, su capacidad para dar instrucciones, como hace el seguimiento a la asignatura, su disponibilidad para resolver dudas, su atención al curso. La representación respecto a su vivencia la construyen en función de la práctica del docente y de aspectos que tienen que ver con su desempeño al dirigir la asignatura, por ejemplo hay quienes expresaron que el maestro es bueno porque se ve que le pone ganas a la materia, o porque es responsable y califica a tiempo o da retroalimentación de las actividades.

Por otra parte, lo cognitivo se valoró en términos del razonamiento $(21 \%)$ que utilizan para resolver problemas o sobre el esfuerzo y dedicación que la modalidad les implica. Hay quien dijo desarrollar habilidades más complejas porque al no tener al maestro enfrente para resolver sus dudas, o no tener la información necesaria, tenía que arreglárselas solo para entender el tema y saber aplicarlo, tal como Sánchez y Castro (2013) lo habían mencionado.

Representaciones sociales de estudiantes universitarios acerca del b-learning: un análisis de contenido. Claudia Islas Torres 
Otra de las ocurrencias más notorias fue lo que respecta a la actitud (30\% sobre el total de unidades textuales) que los estudiantes demuestran al participar en una modalidad $b$ learning, así, el 74\% de éstas, se relaciona a la disposición al cambio que manifestaron los entrevistados, tienen apertura a trabajar en línea y saben organizar su tiempo de acuerdo a sus necesidades, aceptan lo que el ambiente les implica y el utilizar tecnologías como mediación de sus acciones.

Sus actitudes también se encaminan a influir en el docente sobre el uso de TIC (10\%), por ejemplo, algunos estudiantes coincidieron en que si ellos ponían la muestra al docente de cómo utilizar una herramienta o se apoyaban de video tutoriales, eso motivaba a que los docentes se actualizarán y utilizarán estos medios, y lo consideraron como algo positivo; sin embargo, de igual manera hubo profesores que fueron completamente negativos y no aceptaron el uso de tecnología. Esta forma de categorizar haciendo referencia a las actitudes coincidió con lo que Llorente (2008) señalaba en su publicación, ya que lo actitudinal es determinante en la respuesta que un estudiante tendrá ante participar o no de forma activa en la modalidad.

En los discursos fue notoria la dependencia del estudiante hacia el docente (16\%), ya que coincidieron en que prefieren esperar la indicación de éste para actuar, pocos tienen actitudes de iniciativa.

Por lo que respecta a la categoría aprendizaje, solo el $18 \%$ del total de las unidades textuales hacen referencia a éste, de ese 18 el 34\% refirió detalles de autogestión, de cómo a partir de sus necesidades de conocimiento los estudiantes buscan la manera de hacerse de contenidos, ya sea de forma individual o colectiva, se ayudan entre ellos, se comparten información a través de redes sociales o correo electrónico, arman sus propios materiales y aplican sus estrategias de acuerdo a lo que necesiten. En sus representaciones muy pocos hablan del aprendizaje significativo (9\%) pareciera que aunque este es un fin, para ellos es algo irrelevante, se preocupan más por las formas que deben cumplir que por los conocimientos que puedan adquirir y construir a partir de la interacción y colaboración con sus docentes.

Lo que la tecnología les representa (18\% sobre el total de unidades textuales) está relacionado a las habilidades tecnológicas que desarrollan, al uso y aplicación de redes sociales, a su capacidad para administrar información y decidir cual les es útil o no; también hablan sobre como emplean las herramientas web 2.0 y los dispositivos móviles, que les permiten socializar contenidos y conocimientos, independientemente de utilizar una plataforma o no. En este sentido, comparten información a través de grupos cerrados en redes sociales como Facebook, grupos a los que los docentes no tienen acceso, pero que para los estudiantes es una forma de estar en contacto y apoyarse a lo largo del semestre, su tipo de comunicaciones corresponden a interpretaciones sobre las instrucciones del docente, fechas en que deben cumplir con las actividades, críticas sobre los docentes, e inclusive acuerdos para posponer entregas u otras acciones que tienen relación directa con la clase.

Por último, las formas de comunicación que se posibilitan a través del b-learning en las representaciones de los estudiantes significan un $16 \%$ de las unidades textuales, le dan mayor importancia a la retroalimentación del docente refiriéndose a lo oportuno de ésta, y al contenido de la misma; por ejemplo la retroalimentación está valorada en función de la resolución de dudas sobre las actividades que deben realizar. Poco hablan de una retroalimentación constructiva que les ayude a la mejora de su aprendizaje.

Representaciones sociales de estudiantes universitarios acerca del b-learning: un análisis de contenido. 
La comunicación también les representa el intercambio de opiniones entre compañeros, la negociación con el docente sobre todo en términos de entrega de tareas o fechas a cumplir, y la interpretación de instrucciones.

Como puede observarse en la descripción de resultados, las representaciones construidas por los estudiantes pueden ser multifactoriales, sus expresiones son la reconstrucción de lo que sucede y lo que relacionan con una realidad compuesta de diversos elementos que le dan explicación a la cotidianidad de sus clases, sus percepciones, creencias o actitudes son influyentes y determinantes en el desarrollo de sus acciones.

Las representaciones tienen una gran carga de subjetividad, sin embargo, pueden explicarse a partir de las coincidencias y número de ocurrencias en los discursos, de esta forma se da objetivación a lo que se expresa por lo que pueden construirse significados sociales que dan nombre a las cosas y a las personas.

Durante el proceso de análisis se encontraron elementos repetitivos que aparecían en la información tales como ...depende del maestro que te de la clase..., o ... las experiencias han sido buenas y malas..., ...las tecnologías son buenas pero..., esta revisión de elementos fue determinante para la organización de la información. En la figura 2 se expone un modelo de voces que concentra algunos de los comentarios emitidos por los estudiantes y cómo estos en conjunto explican sus representaciones.

Las representaciones construidas por los estudiantes acercan a una realidad multifactorial que caracteriza la dinámica de un ambiente b-learning, y que comprendidas desde esa integridad llevan a deducir los beneficios o desventajas que se visualizan, así como la identificación de la práctica del docente y alumnos.

\section{Conclusiones}

El objetivo de conocer las representaciones que estudiantes universitarios construyen a partir de su vivencia cursando asignaturas de licenciatura en esta modalidad fue logrado tras el ejercicio de codificación e interpretación; los significados inferidos desde los discursos analizados constituyen un aporte más al conocimiento producido en este ámbito y aunado a lo anterior, el referente epistemológico utilizado para el acercamiento al fenómeno (las Representaciones Sociales) permitió apreciar el alcance explicativo que significa acercarse a la experiencia, conciencia y significados de las interrelaciones del estudiante con el contexto en que vive.

El enfoque metodológico aplicado a esta investigación permitió determinar lo que para los estudiantes representa participar en asignaturas que se dirigen bajo la perspectiva de una modalidad b-learning, ya que a través del cuidadoso análisis de la información, se identificaron las verbalizaciones que denotaban lo que para ellos debía ser o no una modalidad mixta valorándola desde sus vivencias, concepciones que a su vez fueron aspecto clave para determinar su actuar durante el curso.

El tomar como fundamento esta teoría permitió tener una visión genérica sobre el fenómeno estudiado además de conocer las relaciones de los elementos que lo componen. Asimismo, posibilitó poner al alcance de la comunidad los resultados de una investigación con un alto contenido subjetivo que a través de la categorización y representación del modelo de voces devela los aspectos involucrados en las representaciones que los estudiantes construyen. El análisis llevado a cabo, nos condujo

Representaciones sociales de estudiantes universitarios acerca del b-learning: un análisis de contenido. Claudia Islas Torres 
a establecer ciertas conclusiones respecto a lo que para los estudiantes representan sus vivencias al cursar asignaturas en modalidad b-learning, desde su perspectiva esta opción de formación es viable, la valoran así por lo que les implica en términos de organización de tiempo, uso de tecnologías o colaboración entre compañeros, autogestión de su aprendizaje y las habilidades que desarrollan para desenvolverse de forma adecuada en el curso, aún a pesar de que anteponen su percepción sobre el docente para poder tomar decisiones sobre su actuar.

Para los estudiantes la modalidad implica desarrollar habilidades que en varios casos les ayudan a subsanar las carencias que desde su perspectiva el docente no puede cubrir. Sus representaciones también se construyen en función de su percepción sobre el profesor, las aptitudes o competencias de éste y el tipo de retroalimentación que reciben de él. Las tecnologías aparecen como indispensables en su actuar porque entre otras cosas les ayudan a ser autónomos y así complementar su proceso de formación.

El ejercicio de abordar esta investigación desde la Teoría de las Representaciones Sociales fue un reto para quien aquí escribe, puesto que es necesario detenerse a contemplar la realidad desde otra perspectiva dejando de lado los prejuicios que sobre el tema se tenían, dando paso a escuchar la voz de los estudiantes y lo que para ellos resulta significante. Esto aunado a la revisión exhaustiva de las experiencias expresadas, proceso que exige un alto nivel de abstracción para llegar a una definición de categorías que dejarían en claro el sentido subjetivo de lo que los estudiantes querían transmitir; además de cuidar que aunque el discurso fuera dividido en partes no se perdiera el contexto de las frases claves o significativas en función de la experiencia tras la cual fueron dichas.

Este fundamento teórico aunado al método de comparación constante de la Teoría Fundamentada y la técnica de análisis de contenido, permitió corroborar la similitud de los discursos en cuanto a la representación que construyen los estudiantes, sintetizando sus explicaciones y en consecuencia, haciendo referencia a un tipo específico de conocimiento que juega un papel crucial sobre como ellos piensan y organizan lo que hacen.

Las representaciones de los estudiantes hacen notar aquello que sucede en el entorno de formación y que para ellos está fuera de lo habitual, o de lo que esperarían como la mejor manera de llevar a cabo su proceso de aprendizaje, encuentran la forma de suplir las carencias de las asignaturas o por el contrario le dedican mayor empeño para aprender lo que les es importante, en este sentido sus representaciones sirven para comprender, actuar u orientarse en el ambiente de aprendizaje.

Representaciones sociales de estudiantes universitarios acerca del b-learning: un análisis de contenido. 
... si el profesor no nos imparte bien la materia, no es que no nos guste es que no nos desempeñamos bien... (P1. 32)

al fin de cuentas si termina influyendo en uno, si termina influyente en tu aprendizaje, en tu forma de aprender... (P6, 29)

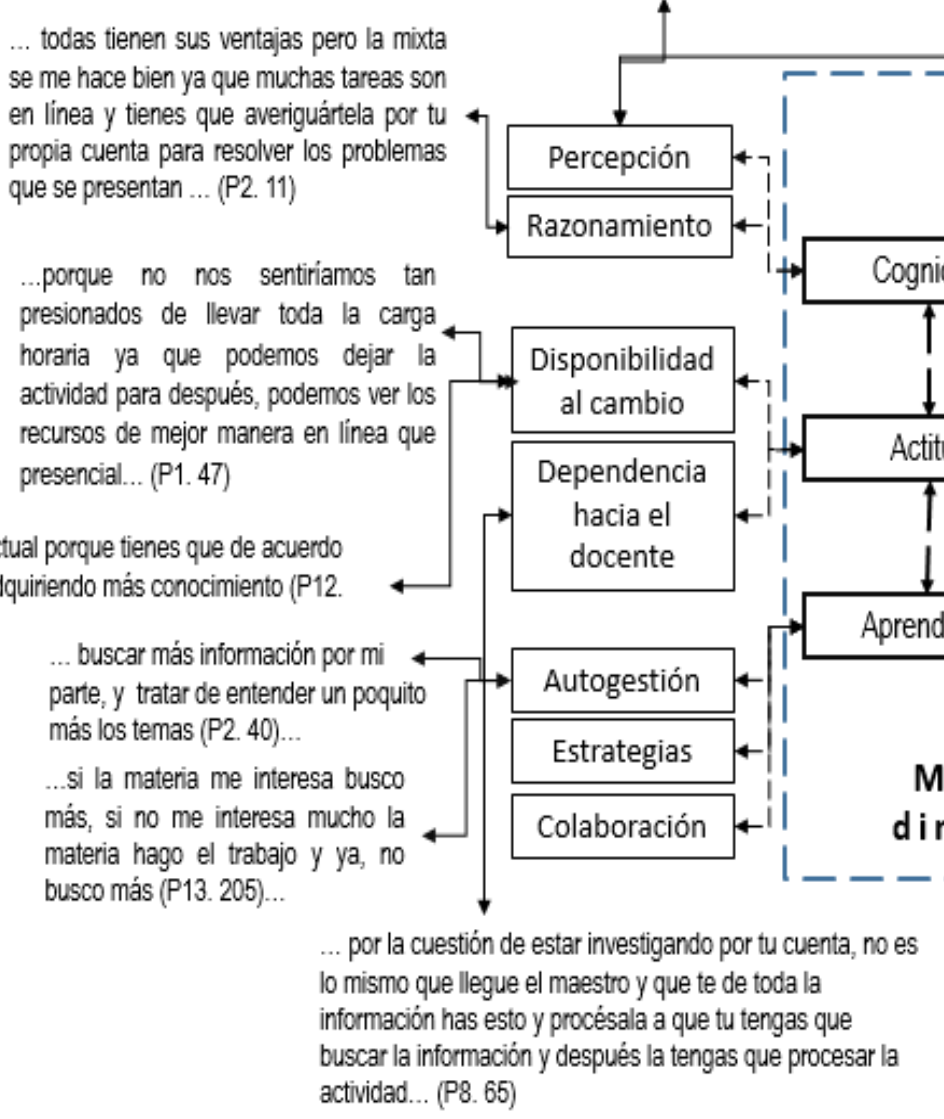
Acción social posibilitada por la modalidad uno mismo va aprendiendo por que si se da en la clase la teoria uno también investiga para realizar las actividades, tiene sus beneficios no se vas acomodando tus cosas para que aproveches ese tiempo de revisar en internet ya casi siempre todas las actividades ocupamos de nuestra computadora y si me gusta esta modalidad... (P12. 51) en el que estamos casi todo el tiempo conectado y si les pregunto sobre alguna actividad o tarea, 0 alguna inquietud que tenga... (P10.18)

es más esfuerzo intelectual porque tienes que de acluedd a las actividades vas adquiriendo más conocimiento ( $\mathrm{P} 12$ 61)

Figura 2. Modelo de voces. Fuente: Creación propia 
En el contenido de sus discursos se encuentran expresiones de valores, actitudes, creencias u opiniones que son compartidas por la mayoría de los estudiantes y que sin duda marcan su actuar.

Asimismo, el enfoque metodológico utilizado permitió conocer y dar cabida a los diferentes puntos de vista que surgían en los discursos de los estudiantes, dando sentido a los sucesos cotidianos que pudieran resultar confusos y que tras el análisis adquirieron significatividad.

El referente epistémico utilizado para esta investigación así como el método y la técnica de análisis de contenido, permitió visualizar su potencial para aplicarlo a trabajos futuros relacionados a la significación de ésta modalidad, actualmente ha servido para estructurar un proyecto de propósitos similares dirigido a los docentes, con la intención de conocer sus representaciones respecto a la implementación de esta modalidad en sus prácticas de enseñanza.

Redacción concluida: 22 de abril de 2016

Fecha de aprobación: 19 de octubre de 2016

Fecha de publicación: 15 de noviembre de 2016

Islas, C. (2016). Representaciones sociales de estudiantes universitarios acerca del b-learning: un análisis de contenido. RED. Revista de Educación a Distancia. 51(6). Consultado el (dd/mm/aaaa) en http://www.um.es/

\section{Bibliografía}

Abric, J. (1994). Prácticas sociales y representaciones. México. Ediciones Coyoacán.

Arras, A.M., Torres C. A., y García-Valcárcel, A. (2011). Competencias en Tecnologías de Información y Comunicación (TIC) de los estudiantes universitarios. Revista Latina de Comunicación Social, 66, 1-23, doi:10.4185/RLCS-66-2011-927-130-152

Ávila-Fajardo, G., y Riascos-Erazo S. (2013). Propuesta para la mediación del impacto de las TIC en la enseñanza universitaria. Educación y Educadores. Recuperado de http://educacionyeducadores.unisabana.edu.co/index.php/eye/article/view/1835/24 $\underline{13}$

Bartolomé, A. (2004). Blended Learning. Conceptos básicos. Pixel-Bit. Revista de medios $y \quad$ educación, 23, 7-20, Recuperado de http://www.lmi.ub.es/personal/bartolome/articuloshtml/04_blended_learning/docu mentacion/1_bartolome.pdf

Campo-Redondo, M., y Labarca, C. (2009). La teoría fundamentada en el estudio empírico de las representaciones sociales: un caso sobre el rol orientador del docente. Opción, 60, 41-54.

Carcaño, A., y Torres C. A. (2013). Una experiencia de aprendizaje en línea sobre el tema del Derecho de Acceso a la Información Gubernamental. Tecnologías y aprendizaje Avances en Iberoamérica, (2), 204-209. Cancún Quintana Roo. Universidad Tecnológica de Cancún.

Representaciones sociales de estudiantes universitarios acerca del b-learning: un análisis de contenido. 
Castañeda, A. Y., y López R. (2014). Lectura y escritura académica en inglés bajo la modalidad de aprendizaje mixto (b-learning). Saber, 26 (2), 202-209. Recuperado de: http://www.redalyc.org/pdf/4277/427739467014.pdf

Chiecher, A., Donolo, D., y Rinaudo, M. C. (2010). Estudiantes universitarios frente al aprendizaje mediado por TIC. Impacto de la propuesta sobre los perfiles motivacionales y las percepciones del curso. Revista Iberoamericana CTS, 1-12, Recuperado de http://www.revistacts.net/files/Portafolio/chiecher_edit.pdf

Chourio, N. (2012). Teoría de las representaciones sociales: discusión epistemológica y metodológica. Estudios Culturales. núm. 10. Recuperado de http://servicio.bc.uc.edu.ve/multidisciplinarias/estudios_culturales/num10/art23.pd $\underline{\mathrm{f}}$

Claro, M. (2010). Impacto de las TIC en los aprendizajes de los estudiantes. Estado del arte. CEPAL. Recuperado de http://www.eclac.org/publicaciones/xml/7/40947/dpimpacto-tics-aprendizaje.pdf

Esparaza, M., Salinas, V., y Glasserman L. D. (2015). La gestión del aprendizaje en la modalidad b-learning frente a la modalidad presencial en la enseñanza de la gramática inglesa. Apertura, 7(2). Recuperado de http://www.redalyc.org/pdf/688/68842702001.pdf

Ferreiro, R., y DeNapoli, A. J. (2006). Un concepto clave para aplicar exitosamente las tecnologías de la educación: los nuevos ambientes de aprendizaje. Revista panamericana de pedagogía. 121-154, Recuperado de http://web.a.ebscohost.com/ehost/pdfviewer/pdfviewer?sid=4b723185-314e-453d8199-4f0abd4575bb\%40sessionmgr4005\&vid=1\&hid=4112

Garrison, R., y Kanuka, H. (2004). Blended Learning: Uncovering its transformative potential in higher education. The Internet and Higher Education, 7 (2), 95-105. doi:doi:10.1016/j.iheduc.2004.02.001

Hernández, R., Fernández, C., y Baptista P. (2010). Metodología de la Investigación. México. McGrawHill.

Hinojo, F., Aznar, I., y Cáseres, M. P. (2009). Percepciones del alumnado sobre blended learning en la universidad. Comunicar. Revista científica Iberoamericana de Comunicación y Edcación, XVII (32), 165-174. doi:http://dx.doi.org/10.3916/C37-2011-02-06

Islas, C. (2014). El b-learning: un acercamiento al estado del conocimiento en Iberoamérica 2003-2013. Apertura, 6(1), 86-97.

Islas, C. (2015). La práctica del estudiante en el b-learning: una observación sistémica. REIRE. Revista d'Innovació $i$ Recerca en Educació 8(2), 43-61. doi:10.1344/reire2015.8.2823

Kvale, S. (2011). Las entrevistas en investigación cualitativa. Madrid. Morata.

Llorente, M. (2008). Actitudes de los alumnos universitarios en procesos de formación blended learning. Revista internacional de Ciencias Sociales y Humanidades, XVIII (2), 91-111.

Representaciones sociales de estudiantes universitarios acerca del b-learning: un análisis de contenido. 
Lobato, C., del Castillo, L., y Arbizu, F. (2005). Las representaciones de la tutoría universitaria en profesores y estudiantes: un estudio de caso. International Journal of Psychology and Psychological Therapy, 5 (2), 148-168.

López, F. (2002). El análisis de contenido como método de investigación. Revista de educación, XXI (4), 67-169.

Materán, A. (2008). Las representaciones sociales: un referente teórico para la investigación educativa. Geoenseñanza, 13(2), 243-248. Recuperado de http://www.redalyc.org/articulo.oa?id=36021230010

Miyazoe, T., y Anderson, T. (2010). Empirical research on learner's perceptions: Interaction Equivalency Theorem in Blended Learning. European Journal of Open, Distance and E-Learning, Recuperado de http://www.eurodl.org/materials/contrib/2010/Miyazoe_Anderson.pdf

Mora, M. (2002). La teoría de las representaciones de Serge Moscovici. Athenea Digital, 2, Recuperado de http://www.raco.cat/index.php/Athenea/article/viewFile/34106/33945

Mortis, S., Hierro, E., García, R., y Manig, A., (2015). La modalidad mixta: un estudio sobre los significados de los estudiantes universitarios. Innovación educativa, 15 (68) Recuperado de http://www.innovacion.ipn.mx/Revistas/Documents/Revistas2015/I-E-68/6-IE-68.pdf

Ojeda, K., Uicab, R., y Aguilar, R. (2011). Curso en línea basado en el diseño instruccional para la enseñanza del cálculo diferencial. Diseño y evaluación de contenidos digitales para la educación. 168-173. Motul Yucatán. Instituto Tecnológico Superior de Motul.

Peñalosa, E., García, C., Martínez, R., y Rojas, G. (2010). Modelo estratégico de comunicación educativa para entornos mixtos de aprendizaje: estudio piloto. PixelBit. Revista de medios y educación, 37, 43-55.

Peres, P., Lima, L., y Lima, V. (2014). B-learning Quality: Dimensions, Criteria and Pedagogical Approach. European Journal of Open Distance and E-learning. Recuperado de http://www.eurodl.org/?p=currentyarticle $=609$

Pulido, O., y Pulido, B. (2012). Diseño de un ambiente b-learning apoyado en estrategias de aprendizaje autorregulado para el estudio de la derivada. Revista EDUCyT. vol. Extraordinario, 198-212. Recuperado de http://revistalenguaje.univalle.edu.co/index.php/educyt/article/view/2027/1952

Rama, C. (2007). La despresencialización de la educación superior en América Latina. ¿Tema de calidad, de cobertura, de internacionalización o de financiamiento?. Apertura. 7(6), 32-49.

Ruíz, C. (2008). El blended learning evaluación de una experiencia de aprendizaje. Revista de Investigación y Posgrado, 23(1), 11-36.

Sánchez, A., y Castro, D. (2013). Cerrando la brecha entre nativos e inmigrantes digitales a través de las competencias informáticas e informacionales. Apertura. $5(2)$, Recuperado de http://www.udgvirtual.udg.mx/apertura/index.php/apertura/article/view/413/334

Representaciones sociales de estudiantes universitarios acerca del b-learning: un análisis de contenido. 
Silvio, J. (sf). Tendencias de la Educación Superior Virtual en América Latina y el Caribe. IESALC. Recuperado de http://www.schoolofed.nova.edu/dll/spanish/modulos/vision/Silvio_Tendencias_E AD_AL.pdf

Strauss , A., y Corbin, J. (2002). Bases de la investigación cualitativa. Técnicas y procedimientos para desarrollar la teoría fundamentada. Antioquia. Universidad de Antioquia

Tello, E.(2008). Las tecnologías de la información y comunicaciones (TIC) y la brecha digital: su impacto en la sociedad de México. Revista de Universidad y Sociedad del Conocimiento, 4(2), 1-8, Recuperado de http://www.uoc.edu/rusc/4/2/dt/esp/tello.pdf

Turpo, O. (2012). La modalidad educativa Blended Learning en las universidades de la Iberoamérica: análisis y perspectivas de desarrollo. Educar, 48(1), 123-147. Recuperado de http://www.raco.cat/index.php/Educar/article/view/252995

Valiathan, P. (2002). Blended Learning Models. Association for talent development. Recuperado de http://www.astd.org/LC/2002/0802_valiathan.htm

Vasileiou, I. (2009). Blended learning: the transformation of higher education curriculum. Open Education: The Journal for Open y Distance Education y Educ, 5, Recuperado de http://connection.ebscohost.com/c/articles/69931820/blendedlearning-transformation-higher-education-curriculum

Villanueva, G., y De la Luz, M. (2010). e-competencias: nuevas habilidades del estudiante en la era de la educación, la globalidad y la generación del conocimiento. Signo y pensamiento, 56, 124-138.

Viñas, M. (2014). TotemGuard, Recuperado de: http://cursoticeducadores.com/ebookcompetencias-digitales-

blog.pdf?inf_contact_key=7b2df2296a82f5910679963ad6c951b5d868cfd58775b5 $\underline{2 \mathrm{a} 222 \mathrm{f} 7 \mathrm{bd} 710 \mathrm{ff} 40 \mathrm{cc}}$

Representaciones sociales de estudiantes universitarios acerca del b-learning: un análisis de contenido. 